\title{
Sinusoidal: An Exploration of Mathematics and Movement
}

\section{Caroline Grace Lockwood}

University of Florida

Faculty mentor: Elizabeth Johnson, School of Theatre and Dance

\begin{abstract}
Mathematic theory often takes place in two-dimensional forms. Whether hand-drawn graphs, or threedimensional models on a screen, the presentation of the subject often lacks the length, breadth, and depth it deserves. Through four phases of research, I explored how one can model mathematic concepts through movement and music, both demonstrably and abstractly. I began my research by basing small dance/movement phrases in simple trigonometric concepts and giving my dancers tasks exploring related subjects. I then examined how other choreographers, both past and present, employed mathematic approaches in their work. To accompany my choreographic work and complete my project, I created a musical score using only single-tone sine wave frequencies. Finally, I interviewed my dancers, as well as other dancers and engineers, to understand how the piece was perceived as both a work of art and an informative display. Through my research, I hope to further the embodied and aesthetic understanding of applied simple and complex mathematic concepts.
\end{abstract}

Keywords: dance, mathematics, trigonometry

\section{Introduction}

Aiming to challenge norms of modeling mathematic and scientific concepts through movement experimentation, I explored ways in which the body can personify concepts of trigonometry as well as the basics of neural networks for artificial intelligence. I feel this work is important to the future understanding of breakthrough ideas and technologies in the fields of science, technology, engineering, and mathematics. This work enlarges and enlivens science, mathematics, and technology at microscopic, macroscopic, and theoretical levels. Embodied processes can aid the inventors' understanding by placing themselves inside two- or three-dimensional models; the final product of such research processes can also break down complex problems and present them in forms that are accessible to a general audience.

I began this journey two years ago in Physics with Calculus I after hearing my professor say the word "sinusoidal." I hated the word but realized its underlying principles could potentially inspire substantial movement. I also felt I could begin to combine my two majors in the arts and sciences, answering the question, "What are you going to do with those two degrees?" 


\section{Discussion of Resources}

There were three main influences in my research: trigonometry, neural networks, and other choreographers. As tools for my work, I used trigonometry to generate movement material, neural networks to inform the narrative of my piece, and work from other choreographers whose compositions have employed mathematic concepts as inspiration.

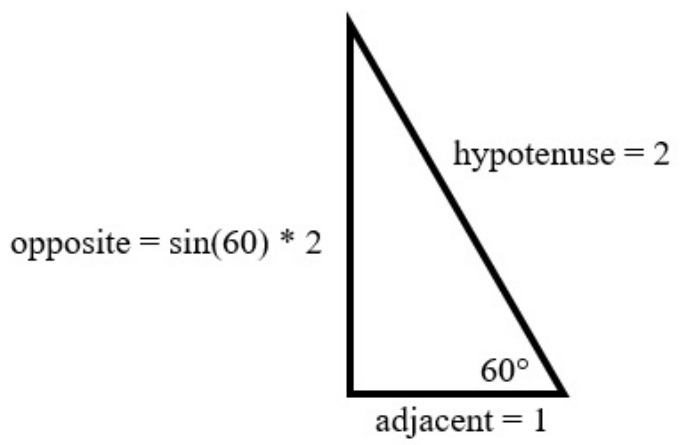

Figure 1. Example of right triangle

\section{Trigonometry}

The trigonometric concepts I used were the number represented by pi, "sohcahtoa," and the graphs of sine, cosine, and tangent. $\mathrm{Pi}$ is an irrational number, meaning it is a number that cannot be expressed as a fraction of two integers and therefore does not terminate or repeat after the decimal point. Pi is crucial for equations involving circles. The abbreviation "sohcahtoa" stands for sine equals opposite over hypotenuse, cosine equals adjacent over hypotenuse, and tangent equals opposite over adjacent. This method is used to find missing side lengths or angles within a right triangle. An example of this would be, as shown above in Figure 1, $\cos (60)=1 / 2$.

The concept of sine, cosine, and tangent originated from a unit circle - a circle with a radius of 1. Originally intended by Gupta period Indian astronomers to find the relationship between a

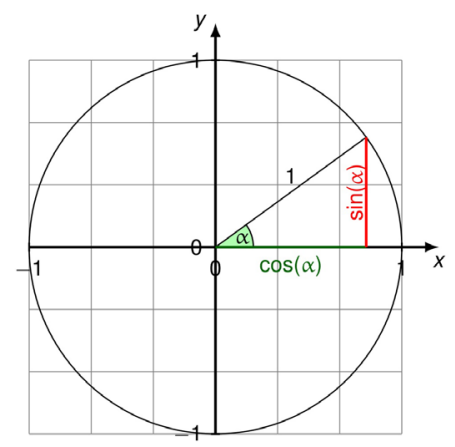

Figure 2. Unit circle (Thoma, 2015) 
chord in a circle and the angle subtended within, the concept of splitting the chord in half gave mathematics the unit circle we know today, as depicted below in Figure 2 (Clark, 2018).

The graph depicting the value of sine as a function of the angle at the origin reveals a repetitive wavelike pattern, shown in Figure 3. A similar pattern emerges for cosine.

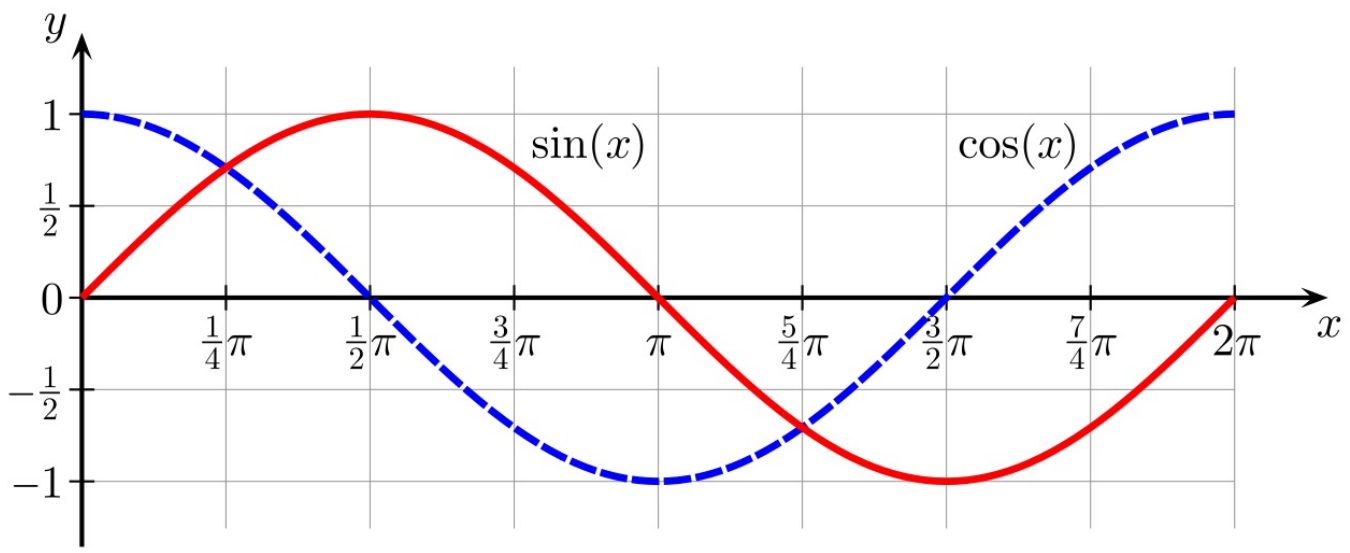

Figure 3. Graph of sine and cosine (Geek3, 2010)

Tangent is the ratio of the opposite side (sine) of the triangle over the adjacent side (cosine). Because the value of cosine equals zero at $\pi / 2$ and $3 \pi / 2$, the denominator of this ratio is zero, therefore creating an asymptote (a line that the graph approaches, but does not touch, as it goes toward infinity) in the tangent graph. The tangent equation creates a similar wavelike pattern, but with a repeated break in the graph, as seen in Figure 4.

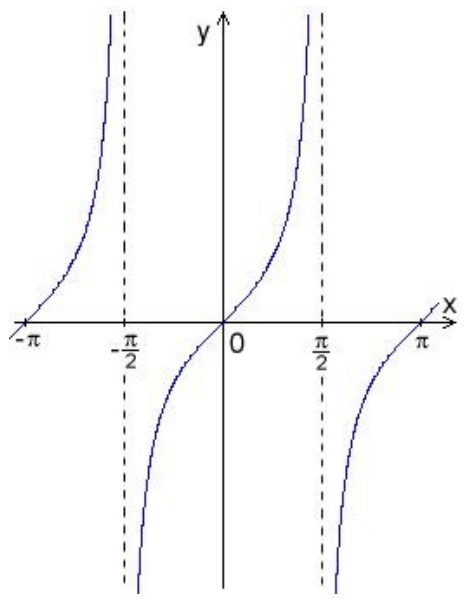

Figure 4. Graph of tangent (Pajs, 2007) 
The word sine came out of a mistranslation across Asia and Europe. The chord in India was referred to as "jyā," meaning "bowstring." A synonym for the word, "jīva," became associated with both the chord and the function of sine. Jīva became "jiba" when translated from Sanskrit to Arabic in Persia. Jiba then became "jb" when abbreviated in Arabic. Europeans then translated jb into Latin using a word they knew, "jayb" in Arabic or "sinus" in Latin. Sinus later became "sine" as it was translated into English. Ultimately, the name we use for these functions has no true meaning (Clark, 2018).

\section{Neural Networks}

The other side of mathematics that informed my work came through the concept of neural networks used in machine learning. At its core, the idea is based on how humans learn. Instead of a human writing code that explicitly tells the computer what to do, the computer uses observation to inform its decisions. The layered approach in learning to understand handwritten numbers, depicted in Figure 5, relies on the concept that activations at one layer determine the activations at the next layer (Sanderson, 2017). These activations are determined by a weighted sum commonly put into a sigmoid function, $\frac{1}{1+e^{-x}}$, which limits the next layer's activation to lie between 0 and 1 . Sine and cosine are similar in that they limit outputs to fall between -1 and 1 . The goal of each layer is to group pixels into small edges, then parts of a number, such as loops and lines, and finally combine those to determine what number is being presented.

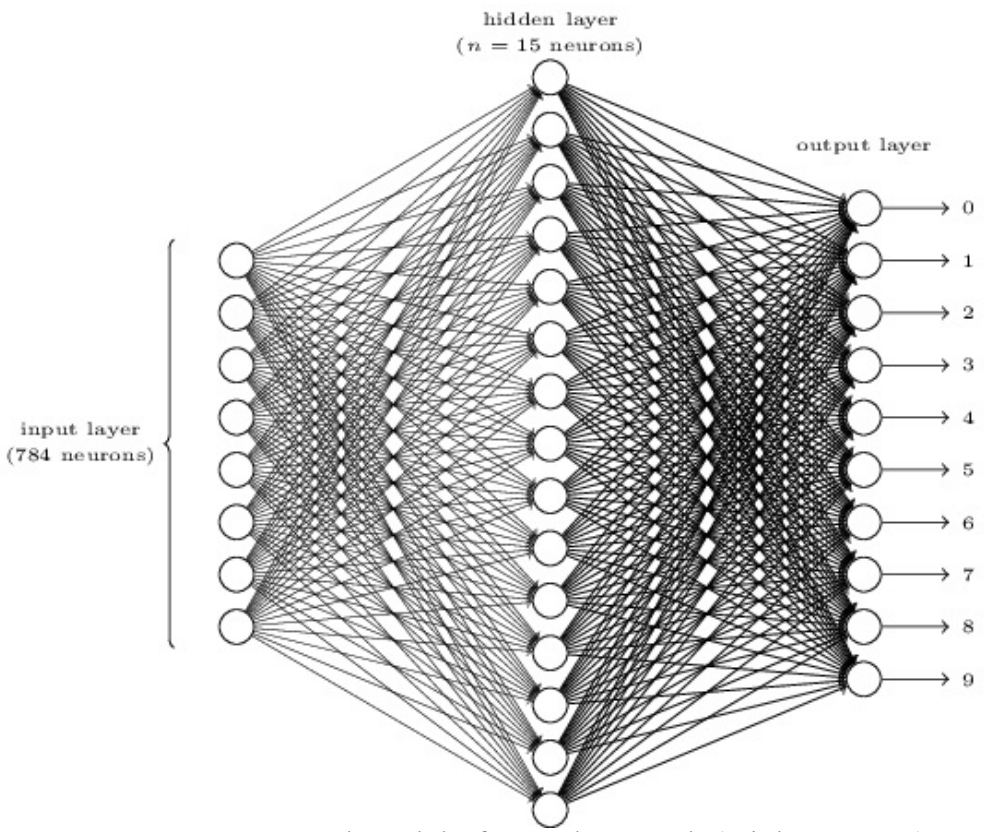

Figure 5. Layered model of neural network (Nielsen, 2015) 


\section{Other Choreographers}

The final portion of resources came from researching choreographers who have employed mathematic concepts in their works, either as a tool for creation or as a concept for presentation. Carl Flink, Artistic Director of Black Label Movement, collaborates closely with Biomedical Engineer David Odde to model complex science projects through movement in a process called "Bodystorming" ("David Odde," n.d.). In this process, Flink and Odde, along with the dancers, design tasks closely related to the action of the concepts Odde is exploring in biomedical engineering. These tasks range from running into one another, simulating collisions within a cell, and dancers wandering around the room with their eyes closed and hands outstretched waiting to find a connection (Patel, 2012). I was able to experience this process first-hand in 2017 when Flink taught classes at the University of Florida.

In her composition, The Fugue, as an engine to create material, Twyla Tharp used a process similar to that of translating a graph in mathematics (Tharp \& Reiter, 2006). Tharp took three steps forward, then three steps back; take a graph, then invert it by adding a negative symbol to the equation. She took three steps to the left, then three steps to the right; add or subtract to the original value of $\mathrm{x}$.

Barbara Dilley, who worked heavily in improvisation in the 1970s defined space for her dancers using a grid. In "The Grid" exercise, the instruction given to the improvisers is to travel along a grid, much like graph paper (Buckwalter, 2011). Often the improvisers are told to start with five simple movements: standing, walking, turning, arm gestures, and crawling. While confining at first, dancers soon realize the grid provides connecting points between one another and opportunities grow from there. "The Grid" can be expanded to use diagonals and even circular grids, which can be found in polar graphs in mathematics.

\section{Scope of Project}

I entered this creative process with the sole focus of embodying sine and cosine through movement. I felt this was a niche enough topic within mathematics to limit the scope to a manageable position, while still providing plenty of concepts from simple mathematics to physics. After spending several weeks working on translating the basics of trigonometry into movement material I realized within this project, we might not ever get to applications of the formulas like physics. I decided to stick with simple trigonometry for the movement invention. 
One of my original ideas for the piece was a line running the length of the body, from wrist to ankle, to highlight the line of the side of the body. I felt there might be ways to embody the graph of sine by shifting the arms, torso, and legs to create wave-like shapes. The way in which I created this line was by having the dancers each wear a set of elastics: two wristbands, two ankle bands, two long stretches of elastics sewn from the wristband to the ankle band (one on each side), and a waistband to pull the elastics closer into the body, as shown below in Figure 6 .

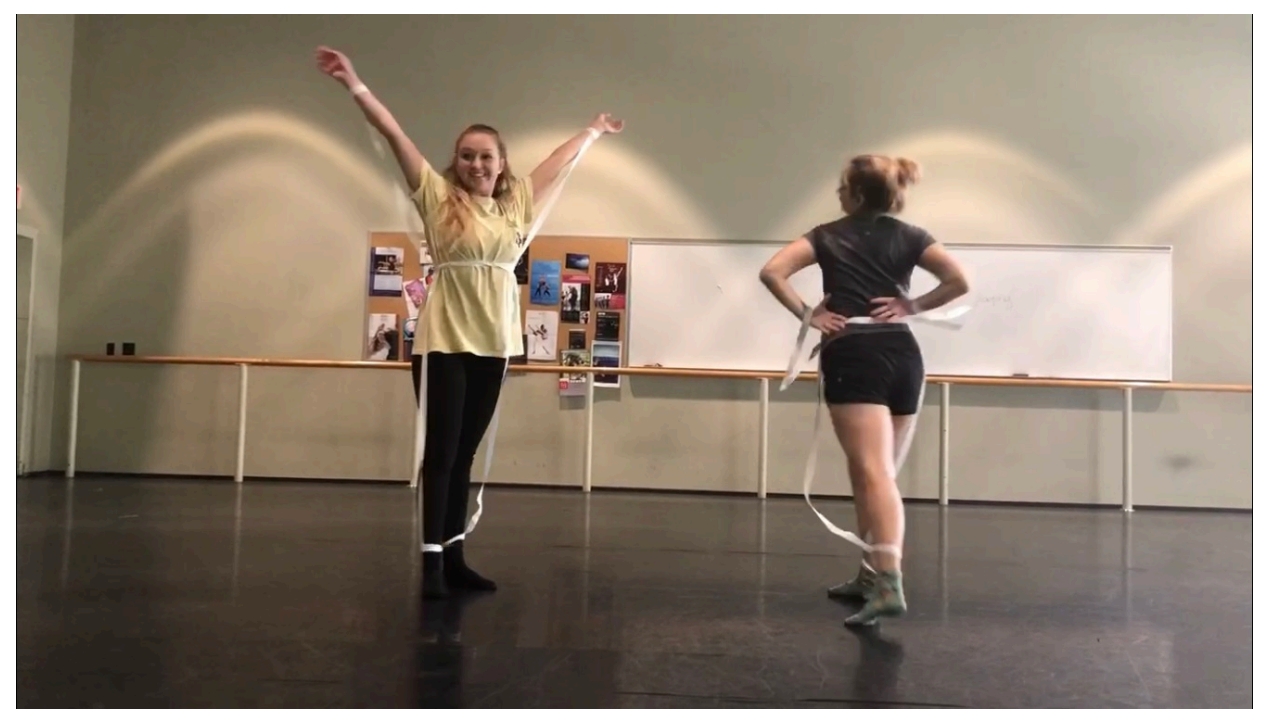

Figure 6. Dancers Olivia Lindfors and Elaina Nelson wearing elastics

During one of the first rehearsals when I asked my dancers to play with them, I assumed they would think about shifting parts of their bodies to make new lines; instead, they began to pull on the elastics, stretching them away from their bodies, and wrapping them around different limbs. We continued to play with this task, coming up with new ways to "tangle" and "untangle" ourselves and each other. The elastics became an extension of the body, helping to create triangles and waves, as well as a means to either support or hinder one another.

During the fall semester in Introduction to Statistical Theory, my professor, Leo Li Duan, introduced neural networks to the class as an application of what we were learning. The topic was interesting, but it wasn't until spring semester that I saw how it fit into my work. By January, I felt we had sufficiently embodied sine and cosine with the movement vocabulary we developed the previous semester, but there was no connecting through line for the piece yet. It occurred to me that the tangling and untangling of the elastics mirrored the image of a layered neural network. We had already been playing with two people tangling up the third person 
shown in Figure 7. I realized that the tangling mimics what the functions in the neural networks were doing to the inputs, so we continued with a concept of two of the dancers (functions) trying to figure out the third dancer (input) and sort her into the correct category.

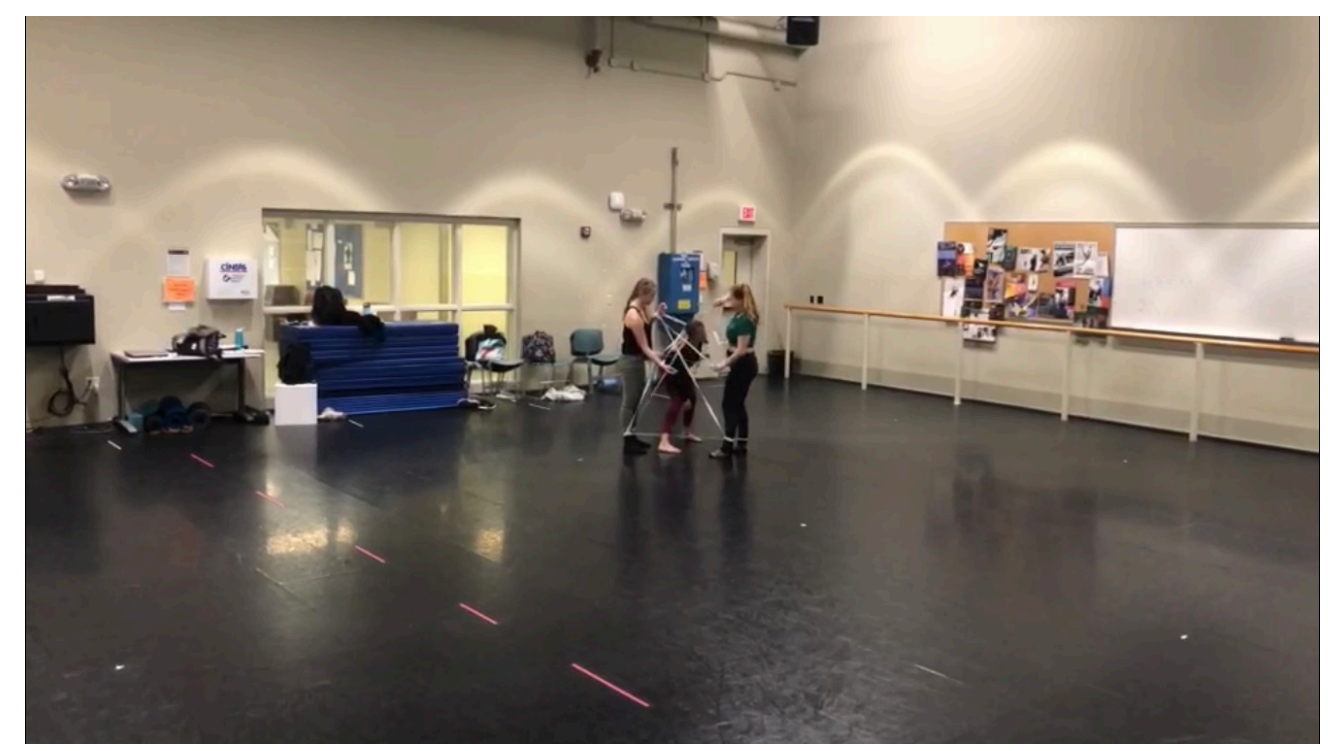

Figure 7. Dancers Olivia Lindfors and Elaina Nelson create a net to tangle Elise Gaudry

To help inform me how to finish the piece, I spoke with my cast about what the work meant to them while they performed it. The consensus was that throughout the composition, the two dancers who represent the functions of sine and cosine are trying to figure out what constant the third dancer is. As the constant goes through the functions, she changes, and they must continue to figure out who she is. In the end, the question became, "Do the functions, and the constant all come together, do they separate, or do they continue as they are?" The dancers agreed from being inside the work, from a physical and emotional understanding; it made sense to come together. With that in mind, the "constant" dancer, Elise Gaudry, asked, "What if the end is tangent?" She pointed out that in the end, everyone knows who they are (sine, cosine, and constant) and when you put all that together, you have tangent $(\tan ($ constant $)=$ $\sin ($ constant $) / \cos ($ constant $))$. The dancers agreed that this motive to become tangent would be that of working together, not resisting. 


\section{Movement Invention}

\section{Phrasework}

To develop movement material, I created a movement for each numeral in the first bit of pi: 3.141592. The characteristics of the numbers I considered were the shape of the numeral and written word, number of beats, sounds, or counts (three might be three beats or one beat for its one syllable), and associations with the number. I then combined the movements for each numeral into the correct order to form pi. After teaching the phrase to my dancers, each dancer came up with a phrase using the same instructions. I felt that beginning from the very basics of the trigonometric properties of sine and cosine would be the most apparent starting point. In later rehearsals, the dancers translated these phrases into movements that reached as far as they could and also in small, gestural phrases. I felt this fit the subject matter of damping of sine wave patterns or decreasing the amplitude of the wave.

The second bit of phrasework I created revolved around the concept of "sohcahtoa" and the history of the word sine. The phrase bends the knees which creates in the body the sides of the triangle needed for sine and simultaneously gestures at the sinus cavity and chest as a nod to the final form of the mistranslated word.

Another section we worked on early on was creating a sine wave with the dancers standing next to one another and then changing levels in a ripple - think "the wave" in a stadium. However, instead of standing from sitting, rather it descends by bending at the knees and going from standing to squatting and back up again. I took gestures and movement material from all four of our "pi phrases" to create this short level changing phrase.

To make the graph of the sine wave more present, I played with strategically placing my dancers in space. Typically, this meant there were two dancers on one side of the room and the third dancer, on the opposite side, would eventually weave through the center of the duet somewhere in the middle of the space, as shown in Figure 8. This traveling formation replicated the wave of a sine graph going from above the $\mathrm{x}$-axis to below the $\mathrm{x}$-axis. 

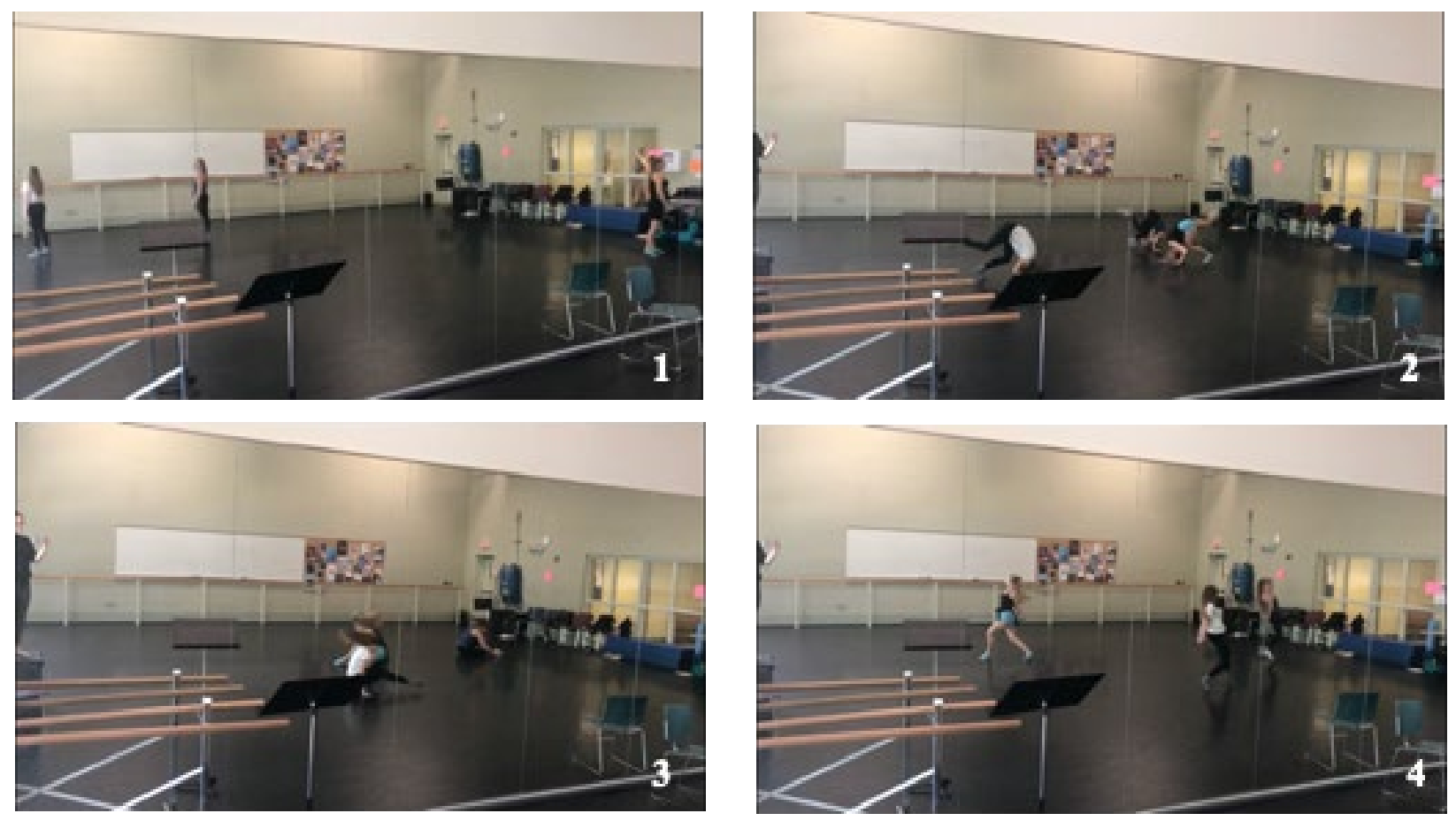

Figure 8. Dancers creating a sine wave in space

\section{Introducing a Prop}

We spent several rehearsals figuring out the mechanics of tangling and untangling oneself and others with elastics as props. Tangling other people while they were doing their movement required intense observation on the "tangler's" part. Co-tangling with someone else required teamwork. Tangling oneself was free-flowing. What was interesting was that when it came time to untangle, the situations that seemed like they might be challenging to escape were the easiest to slip out of and vice versa.

To make the tangling as seamless as possible, we created reproducible tangles for each moment they reappear within the work. The persistent effort to make these tangles quicker and more efficient is similar to the continual learning machines do through neural networks.

We also played with doing phrasework while tangled. The part that ended up in the end product was the level changing phrase. The elastics added an extra layer when the first dancer pulled away from the group, which sent a chain reaction pulling the next dancer, and then the next. 


\section{Other Sources of Invention}

There were some tasks we played with that were edited from the final composition. I gave the dancers improvisational tasks such as "secure the perimeter" (like sine contains data between 1 and -1), do movements in sets of threes (which is close to the value for pi), and always try to be underneath someone (like the hypotenuse in the ratios for sine and cosine).

To make a task out of the mistranslation of the word sine, we spent one rehearsal playing a game of telephone with choreography. I gave one dancer a phrase and told her to do the phrase using her full kinesphere — or the full extent of her range of motion from fingers to toes - for the entirety of the phrase. The first dancer then taught that version of the phrase to the second dancer, who was then instructed to do the transformed phrase traveling through space, following a path as if there was a sine wave drawn on the floor. The second dancer taught the third dancer, who was then instructed to perform the phrase with the quality of a spring (this was at a time when I thought we might be able to incorporate physics concepts).

\section{Music}

Single sound frequencies can be represented as sine waves. I created music for this piece using only these tones. With the software Audacity, I used the "generate tone" tool in various frequencies and altered them using different effects. I watched a video from my rehearsal, one move at a time, and created a sound that matched the movement. For some sections of the dance, I did this for every movement, while for others, I created an overarching soundscape. The tools I used were: sliding shift (which slowly increases the frequency of the tone), phaser (which damps the wave, decreasing the amplitude of the tone), fade in and fade out (which increases or decreases the amplitude linearly), and reverse (which plays the affected tone backward). I also used a tool for generating rhythm tracks creating a steady beat of single tone sine waves.

\section{Audience Response}

As a final step to my research, I conducted interviews with my peers inquiring what concepts they were able to see in the composition. The people I talked to knew what the piece was about, as far as that I was embodying sine and cosine through movement and exploring concepts of machine learning. With that lens, many saw mathematic concepts but were still able to identify other ideas. With more time, I would love to show this work out of context to see if the movement is identifiable on its own. 


\section{Noesha Noel}

Noel is a first-year Mathematics and BFA Dance double major.

The first concept Noel mentioned was that it seemed like when two dancers created a tangled net for the third dancer to pass through, the two dancers on the side represented 1 and -1 in which a sine wave is typically bound. She continued with this concept to say that since that portion of the dance travels down a long diagonal, it seemed like the dancer was travelling from negative infinity to positive infinity on the $\mathrm{x}$-axis, just as a graph of sine would.

Another concept Noel saw was that the dancers who represented the functions moved more quickly when they were closer together and slower when they were further apart. She connected this to the relationship between wavelength and frequency - the shorter the wavelength, or distance between each wave, the higher the frequency, the more repetitions within a certain amount of time.

Noel mentioned that the solo parts in the piece had clear images of sine waves through arm movements as well as angular movements that hinted at the concept of sohcahtoa. She also mentioned that related to sohcahtoa and triangles; it was perfect that there were three dancers for the three sides of a triangle.

\section{Jessica Rivas}

Rivas is a third year BFA Dance major.

Without the details of the mathematic concepts, Rivas identified a storyline about Gaudry finding her place in society without letting society define her. She noted that Lindfors and Nelson were consistent in their movements throughout the piece, while Gaudry had more freedom of movement as if she were trying to find the movement that fit her place in society. Lindfors and Nelson have things tied to them that they try to attach to Gaudry-sometimes Gaudry allows this attachment (finding her place in society), but sometimes she breaks away from the attachment (society does not define her).

After discussing the mathematic concepts, Rivas easily detected triangular poses and the neural network made by an elastic net. Rivas observed two dancers trying to figure out where the third fit in the net. As the choreographer, this was an overarching storyline I was aiming for in this mathematic lens, but Rivas was able to perceive this without mathematics. 


\section{Greg Wiley}

Wiley is a fifth-year Industrial and Systems Engineering major.

Knowing the piece was in general about sine and neural networks, Wiley pinpointed moments throughout the composition he felt symbolized mathematic concepts. He noted the movement at the beginning was stop and go, just as a sine wave goes up, back down, to zero then continues again. He said the ropes symbolized how each period of a sine wave is connected to the next. He noticed several instances of repetition, representing the repetition of sine waves, as well as curvature in the arms, representing the shape of each wave.

In terms of relationships between the dancers, Greg observed that two dancers were always connected while the third kept repeating herself on her own. The pair would pull the soloist in, then take her out, and back in again, showing repetition in their relationship.

The stretching of the elastics suggested the flexibility of functions in math, while the moments when the elastics bound the dancers together illustrated that all mathematic concepts are interconnected.

\section{Conclusion and Future Applications}

When I spoke to my dancers near the end of our creation and rehearsal process, I was pleased to hear them explain how this form of embodiment helped them to understand the concepts further. Gaudry noted that it wasn't a different understanding of the concepts, but a physical connection was made. I feel that this physical understanding is not unlike learning how to manually compute mathematic operations before relying on a calculator or computer software. Because we were able to break down the operations into basic terms and translate them to the basic human understanding of movement, the dancers were able to make connections detailing the parts (or, in this case, people) needed for the math to function.

Moving forward, I hope to use what I learned in this process in applications such as the choreography of coding - how technologies like Apple Watches rely on the programmer's choreographic understanding of human gestures to successfully function. I feel having choreographers who understand both the technology and body language and can integrate the two, can then help with the development of technology for all bodies.

A link to my composition can be found here: https://youtu.be/s5-fyXt4J64. 


\section{Acknowledgments}

I want to thank my mentor, Elizabeth Johnson, for guiding me through this process for the last two semesters. I want to thank my dancers - Elise Gaudry, Olivia Lindfors, and Elaina Nelson-for their trust in me as a first-time choreographer and their creativity in every rehearsal. I want to thank everyone who took the time to give me feedback along the way and the teachers who unknowingly introduced me to concepts that would change the course of this research. Finally, I would like to thank Dr. Donnelly and the University Scholars Program for supporting my research.

\section{References}

Buckwalter, M. (2011). Composing while dancing an improvisers companion. Madison, WI: University of Wisconsin Press.

Clark, S. (2018, January 25). Why sin and cos don't mean anything. Retrieved from https://www.youtube.com/watch?v=AzVL4321EWA

David Odde. (n.d.). Retrieved from https://www.blacklabelmovement.com/david-odde

Elert, G. (1998). Trigonometry - The Physics Hypertextbook. Retrieved March, 2019, from https://physics.info/trigonometry/

Geek3. (2010, July 16). Sine cosine one period [Digital image]. Retrieved March, 2019, from https://commons.wikimedia.org/wiki/File:Sine_cosine_one_period.svg

Mazzoni, D., \& Dannenberg, R. (2000). Audacity (Version 2.3.0) [Computer software]. Retrieved March, 2019 .

Nielsen, M. (2015). Neural Networks and Deep Learning. Retrieved March, 2019, from http://neuralnetworksanddeeplearning.com/chap1.html

Nuye/01asdfg23. (2010, August 25). Music for sine wave. Retrieved January, 2019, from https://www.youtube.com/watch?v=_Sj8p2T6380

Pajs. (2007, May 23). Graf tangens [Digital image]. Retrieved March, 2019, from https://commons.wikimedia.org/wiki/File:Graf_tangens.png

Patel, K. (2012, December 06). Bodystorming- Black Label Movement. Retrieved from https://www.youtube.com/watch?v=wy2UYFgbwxc

Sanderson, G. (2017, October 05). But what *is* a Neural Network? | Deep learning, chapter 1. Retrieved from https://www.youtube.com/watch?v=aircAruvnKk

TED-Ed. (2012, November 28). Dance vs. PowerPoint, a modest proposal - John Bohannon. Retrieved from https://www.youtube.com/watch?time_continue=3\&v=0nqhopRhju4

Tharp, T., \& Reiter, M. (2006). The creative habit learn it and use it for life: A practical guide. New York: Simon \& Schuster. 
Thoma, M. (2015, November 5). Triangle-unit-circle [Digital image]. Retrieved March, 2019, from https://commons.wikimedia.org/wiki/File:Triangle-unit-circle.sv 